\title{
UNIQUENESS RESULTS FOR A CLASS OF HIGHER - ORDER BOUNDARY VALUE PROBLEMS
}

\author{
CRISTIAN-PAUL DANET \\ Department of Applied Mathematics, University of Craiova \\ Al. I. Cuza St., 13, 200585 Craiova, Romania \\ e-mail: cristiandanet@yahoo.com
}

(Received 24 February, 2006; revised 27 April, 2006; accepted 2 May, 2006)

\begin{abstract}
The classical maximum principle is utilized to obtain maximum principles for functionals which are defined on solutions of fourth, sixth and eighthorder elliptic equations. The principles derived lead to uniqueness results.
\end{abstract}

2000 Mathematics Subject Classification. 35J40, 35B50.

1. Introduction. It is well known that every subharmonic function in a bounded domain $\Omega$ (i.e. $\Delta u \geq 0$ in $\Omega$ ) satisfies the classical maximum principle

$$
\max _{\bar{\Omega}} u=\max _{\partial \Omega} u
$$

The subbiharmonic function $u(x)=-x_{1}^{4}-|x|^{2}$ in the ball $\Omega=\left\{\left(x_{1}, \ldots, x_{n}\right)|| x \mid<\right.$ $R$ \} (i.e. $\Delta^{2} u \leq 0$ in $\Omega$ ) shows that there are no classical maximum principles for the biharmonic operator $\Delta^{2} u$ (and for higher-order elliptic operators at all). Still some results can be proven.

The first proof of a maximum principle for an elliptic equation of higher-order was given by Miranda [4]. The Miranda result was extended to semilinear equations by Schaefer [5] and used to deduce the non-existence of solution to certain boundary value problems. An extension of this last maximum principle to a sixth-order equation may be found in [6]. There are other works dealing with maximum principles for fourth and sixth-order equations and their applications (see [1], [2], [3], [7] (Chapter 10), [9] and the references cited therein). A general maximum principle for an equation with constant coefficients of order $2 m$ was established by Tseng and Lin ([8]).

Our purpose here is to deduce some maximum principles for a class of fourth and sixth-order equations which do not appear to be contained in the above mentioned works. A maximum principle for an equation of eighth-order with non-constant coefficients is also presented. From these maximum principles we obtain uniqueness results for a class of higher-order equations in a plane domain $\Omega$. If the domain is on the line, then we briefly indicate some uniqueness results for problems of order $2 m(m \geq 5)$.

2. Maximum principles and uniqueness results. Let $\Omega$ be a bounded domain in the plane with a smooth boundary $\partial \Omega$. We denote partial derivatives $\partial u / \partial x_{i}$ by $u,{ }_{i}$ and use the summation convention, so that, e.g., the square of the gradient of $u$ becomes 
$\left(\partial u / \partial x_{1}\right)^{2}+\left(\partial u / \partial x_{2}\right)^{2}=|\nabla u|^{2}=u,{ }_{i} u,{ }_{i}$. The scalar product of the gradients of two functions, say $u$ and $v$, may be written as $\nabla u \cdot \nabla v=u,{ }_{i} v,{ }_{i}$.

First, we consider a fourth-order equation

$$
\Delta^{2} u-a(x) \Delta u+b(x) u=0 \quad \text { in } \Omega,
$$

where

$$
a>0, \quad \Delta(1 / a) \leq 0
$$

and

$$
b \geq 0
$$

We have the following result.

LEMMA 2.1. If $u \in C^{4}(\Omega) \cap C^{2}(\bar{\Omega})$ is a (classical) solution of (2.1), then the functional $\mathrm{F}_{1}$ given by

$$
\mathrm{F}_{1}=\frac{a(x)}{2} u^{2}+\left(|\nabla u|^{2}-u \Delta u\right)
$$

assumes its maximum value on $\partial \Omega$.

From Lemma 2.1 we now deduce a slight extension of Schaefer's result $(\mathbf{6}$, Theorem 4).

THEOREM 2.1. There is at most one classical solution of the boundary value problem

$$
\left\{\begin{array}{l}
\Delta^{2} u-a(x) \Delta u+b(x) w(u)=f \quad \text { in } \Omega \\
u=g, \quad \frac{\partial u}{\partial n}=h \quad \text { on } \partial \Omega
\end{array}\right.
$$

where $a, b$ satisfy (2.2) and (2.3) and $w$ is a $C^{1}$ function for which bw' $>0$ in $\Omega$.

Here and throughout the paper $\partial / \partial n$ denotes the outward normal derivative operator.

It is quite obvious how Theorem 1 and Theorem 4 in [5] have to be modified to obtain the above stated results. The details are left to the reader.

We now prove a maximum principle for the sixth-order equation

$$
\Delta^{3} u-a(x) \Delta^{2} u+b(x) \Delta u-c(x) u=0 \quad \text { in } \Omega \subset \mathbb{R}^{2},
$$

where

$$
c>0, \quad \Delta(1 / c) \leq 0 .
$$

LEMMA 2.2. Let $u \in C^{6}(\Omega) \cap C^{4}(\bar{\Omega})$ be a (classical) solution of (2.6) and suppose that (2.2), (2.3) and (2.7) are satisfied. Then the functional $\mathrm{F}_{2}$ given by

$$
\mathrm{F}_{2}=\frac{c(x)}{2} u^{2}+\frac{a(x)}{2}(\Delta u)^{2}+|\nabla(\Delta u)|^{2}-\Delta u \Delta^{2} u
$$

assumes its maximum value on $\partial \Omega$. 
Proof. The assumption (2.2) implies that

$$
\begin{aligned}
\Delta\left(c(x) u^{2}\right) / 2 & \geq c(x) u \Delta u+\sum_{i=1}^{2}\left(\frac{1}{\sqrt{c}} c,{ }_{i} u+\sqrt{c} u,{ }_{i}\right)^{2} \\
& \geq c(x) u \Delta u
\end{aligned}
$$

Similarly,

$$
\Delta\left(a(x)(\Delta u)^{2}\right) / 2 \geq a(x) \Delta u \Delta^{2} u
$$

Now

$$
\begin{aligned}
& \Delta\left(|\nabla(\Delta u)|^{2}\right)=2(\Delta u),_{i j}(\Delta u),_{i j}+2 \nabla(\Delta u) \cdot \nabla\left(\Delta^{2} u\right) \\
& \Delta\left(\Delta u \Delta^{2} u\right)=\left(\Delta^{2} u\right)^{2}+2 \nabla(\Delta u) \cdot \nabla\left(\Delta^{2} u\right)+\Delta u \Delta^{3} u
\end{aligned}
$$

Hence

$$
\Delta \mathrm{F}_{2} \geq 2(\Delta u),_{i j}(\Delta u),{ }_{i j}-\left(\Delta^{2} u\right)^{2}+b(x)(\Delta u)^{2} \quad \text { in } \Omega .
$$

Since the inequality

$$
2 v,{ }_{i j} v, i j \geq(\Delta v)^{2}
$$

holds in two dimensions, we obtain

$$
\Delta \mathrm{F}_{2} \geq 0 \text { in } \Omega \text {. }
$$

An immediate consequence of Lemma 2.2 is the following uniqueness result.

THEOREM 2.2. There is at most one classical solution of the boundary value problem

$$
\left\{\begin{array}{l}
\Delta^{3} u-a(x) \Delta^{2} u+b(x) \Delta u-c(x) u=f \quad \text { in } \Omega \\
u=g, \quad \Delta u=h, \quad \frac{\partial(\Delta u)}{\partial n}=i \quad \text { on } \partial \Omega
\end{array}\right.
$$

where $a, b, c$ satisfy (2.2), (2.3) and (2.7).

Proof. Assume that $u_{1}$ and $u_{2}$ are solutions of (2.10) and let $v=u_{1}-u_{2}$. The function $v$ satisfies (2.6) and

$$
v=\Delta v=\frac{\partial(\Delta v)}{\partial n}=0 \quad \text { on } \partial \Omega
$$

Since $\Delta v=0$ on $\partial \Omega$ we obtain that $|\nabla(\Delta v)|^{2}=\left(\frac{\partial(\Delta v)}{\partial n}\right)^{2}$ on $\partial \Omega$.

Hence, by Lemma 2.2 and (2.11),

$$
\mathrm{F}_{2} \leq \max _{\partial \Omega} \mathrm{F}_{2}=0 \quad \text { in } \Omega
$$

Consequently,

$$
-\Delta v \Delta^{2} v \leq 0 \quad \text { in } \Omega
$$


Integrating (2.13) over $\Omega$ and using Green's identity we conclude that

$$
|\nabla(\Delta v)|=0 \quad \text { in } \Omega
$$

and by continuity $\Delta v \equiv 0$ in $\Omega$. Since $v=0$ on $\Omega$ we are led to the conclusion that $v \equiv 0$ in $\Omega$.

Next we deal with classical solutions (i.e. $C^{8}(\Omega) \cap C^{6}(\bar{\Omega})$ ) of

$$
\Delta^{4} u-a(x) \Delta^{3} u+b(x) \Delta^{2} u-c(x) \Delta u+d u=0 \quad \text { in } \Omega \subset \mathbb{R}^{2},
$$

where

$$
d>0 \text {. }
$$

A uniqueness result can be inferred from the following maximum principle

Lemma 2.3. Let u be a classical solution of (2.14). Assume that (2.2), (2.3), (2.7) and (2.15) are satisfied. Then the functional

$$
\mathrm{F}_{3}=\frac{c(x)}{2}(\Delta u)^{2}+\frac{a(x)}{2}\left(\Delta^{2} u\right)^{2}+d\left(|\nabla u|^{2}-u \Delta u\right)+\left|\nabla\left(\Delta^{2} u\right)\right|^{2}-\Delta^{2} u \Delta^{3} u
$$

assumes its maximum value on $\partial \Omega$.

Proof. Using (2.2) and (2.7), we have

$$
\begin{gathered}
\Delta\left(c(x)(\Delta u)^{2}\right) / 2 \geq c(x) \Delta u \Delta^{2} u, \\
\Delta\left(a(x)\left(\Delta^{2} u\right)^{2}\right) / 2 \geq a(x) \Delta^{2} u \Delta^{3} u .
\end{gathered}
$$

By inequality (2.9) we get

$$
\begin{gathered}
d \Delta\left(|\nabla u|^{2}-u \Delta u\right)=d\left(2 u,{ }_{i j} u,,_{i j}-(\Delta u)^{2}-u \Delta^{2} u\right) \geq-d u \Delta^{2} u, \\
\Delta\left(\left|\nabla\left(\Delta^{2} u\right)\right|^{2}-\Delta^{2} u \Delta^{3} u\right)=2\left(\Delta^{2} u\right),{ }_{i j}\left(\Delta^{2} u\right),{ }_{i j}-\left(\Delta^{3} u\right)^{2}-\Delta^{2} u \Delta^{4} u \geq-\Delta^{2} u \Delta^{4} u .
\end{gathered}
$$

Hence from equation (2.14) we obtain

$$
\Delta \mathrm{F}_{3} \geq 0 \quad \text { in } \Omega \text {. }
$$

THEOREM 2.3. There is at most one classical solution of the boundary value problem

$$
\left\{\begin{array}{l}
\Delta^{4} u-a \Delta^{3} u+b(x) \Delta^{2} u-c \Delta u+d u=f \quad \text { in } \Omega \\
u=g, \quad \Delta u=h, \quad \Delta^{2} u=i, \quad \frac{\partial\left(\Delta^{2} u\right)}{\partial n}=j \quad \text { on } \partial \Omega
\end{array}\right.
$$

where a satisfies (2.2), b satisfies (2.3), c satisfies (2.7), $d>0$, and the curvature $k$ of $\partial \Omega$ is positive.

Proof. To establish this result, we suppose that $u_{1}$ and $u_{2}$ are two solutions of (2.17). Defining $v=u_{1}-u_{2}$, we see that $v$ satisfies (2.14) and

$$
v=\Delta v=\Delta^{2} v=\frac{\partial\left(\Delta^{2} v\right)}{\partial n}=0 \quad \text { on } \partial \Omega
$$


Since $v=\Delta^{2} v=0$ on $\partial \Omega$, we obtain

$$
\begin{aligned}
\frac{\partial \mathrm{F}_{3}}{\partial n}= & c \Delta v \frac{\partial(\Delta v)}{\partial n}+a \Delta^{2} v \frac{\partial\left(\Delta^{2} v\right)}{\partial n}+d\left(2 \frac{\partial v}{\partial n} \frac{\partial^{2} v}{\partial n^{2}}-\frac{\partial v}{\partial n} \Delta v-v \frac{\partial(\Delta v)}{\partial n}\right) \\
& +2 \frac{\partial\left(\Delta^{2} v\right)}{\partial n} \frac{\partial^{2}\left(\Delta^{2} v\right)}{\partial n^{2}}-\frac{\partial\left(\Delta^{2} v\right)}{\partial n} \Delta^{3} v-\Delta^{2} v \frac{\partial\left(\Delta^{3} v\right)}{\partial n} \quad \text { on } \partial \Omega .
\end{aligned}
$$

By (2.18) we have

$$
\frac{\partial \mathrm{F}_{3}}{\partial n}=2 d \frac{\partial v}{\partial n} \frac{\partial^{2} v}{\partial n^{2}} \quad \text { on } \partial \Omega
$$

By introducing normal coordinates in the neighbourhood of the boundary, we can write

$$
\Delta v=\frac{\partial^{2} v}{\partial n^{2}}+\frac{\partial^{2} v}{\partial s^{2}}+k \frac{\partial v}{\partial n},
$$

where $\frac{\partial v}{\partial s}$ denotes the tangential derivative of $v$.

Since $v=\Delta v=0$ on $\partial \Omega$, relation (2.20) becomes

$$
\frac{\partial^{2} v}{\partial n^{2}}=-k \frac{\partial v}{\partial n}
$$

which gives

$$
\frac{\partial \mathrm{F}_{3}}{\partial n}=-2 d k\left(\frac{\partial v}{\partial n}\right)^{2} \leq 0 \quad \text { on } \partial \Omega
$$

This contradicts Hopf's lemma at a point $P \in \partial \Omega$, where $\mathrm{F}_{3}\left(\mathrm{~F}_{3} \not \equiv\right.$ constant $)$ assumes its maximum value. Hence $F_{3}$ is constant in $\bar{\Omega}$. Thus $\frac{\partial F_{3}}{\partial n}=0$ on $\partial \Omega$ and consequently $\frac{\partial v}{\partial n}=0$ on $\partial \Omega$. By the boundary conditions it follows that $\mathrm{F}_{3}=0$ in $\bar{\Omega}$. Using Green's identity we arrive at $v=0$ in $\bar{\Omega}$.

Finally we shift our attention from the two dimensional to the one dimensional case and mention the following result ( $\Omega$ denotes an open interval $(\alpha, \beta)$ ).

THEOREM 2.4. There can be at most one classical solution of the problem

$$
\left\{\begin{array}{l}
u^{(2 m)}-d u^{(6)}+c(x) u^{(4)}-b(x) u^{\prime \prime}+a(x) u=f \quad \text { in } \Omega \\
u=g_{1}, u^{\prime \prime}=g_{2}, u^{\prime \prime \prime}=g_{3}, \ldots, u^{(m)}=g_{m} \quad \text { on } \partial \Omega,
\end{array}\right.
$$

where $m \geq 6$ is even, $d \geq 0$ and $b \geq 0, a, c>0,(1 / a)^{\prime \prime},(1 / c)^{\prime \prime} \leq 0$ in $\Omega$.

The result follows since the function

$$
\begin{aligned}
\mathrm{F}_{4}= & u^{\prime \prime} u^{(2 m-2)}-2 u^{\prime \prime \prime} u^{(2 m-3)}+3 u^{(4)} u^{(2 m-4)}-\cdots+(m-3) u^{(m-2)} u^{(m+2)} \\
& -(m-3) u^{(m-1)} u^{(m+1)} / 2-((m-3) / 2+1)\left[\left(u^{(m)}\right)^{2}-u^{(m-1)} u^{(m+1)}\right] \\
& +\left[\left(u^{\prime \prime \prime}\right)^{2}-d u^{\prime \prime} u^{(4)}\right]+c(x)\left(u^{\prime \prime}\right)^{2} / 2+a(x) u^{2} / 2
\end{aligned}
$$

assumes its maximum value on $\partial \Omega$, where $u$ is a solution of

$$
u^{(2 m)}-d u^{(6)}+c(x) u^{(4)}-b(x) u^{\prime \prime}+a(x) u=0 \quad \text { in } \Omega .
$$


Similarly, we can treat the problem

$$
\left\{\begin{array}{l}
u^{(2 m)}+d u^{(6)}-c(x) u^{(4)}+b(x) u^{\prime \prime}-a(x) u=f \quad \text { in } \Omega \\
u=g_{1}, u^{\prime \prime}=g_{2}, u^{\prime \prime \prime}=g_{3}, \ldots, u^{(m)}=g_{m} \quad \text { on } \partial \Omega,
\end{array}\right.
$$

where $m \geq 5$ is odd.

\section{REFERENCES}

1. D. R. Dunninger, Maximum principles for solutions of some fourth order elliptic equations, J. Math. Anal. Appl. 37 (1972), 655-658.

2. V. B. Goyal, Liouville-type results for fourth order elliptic equations, Proc. Roy. Soc. Edinburgh Sect. A 103 (1986), 209-213.

3. S. Goyal and V. B. Goyal, Liouville-type and uniqueness results for a class of sixth-order elliptic equations, J. Math. Anal. Appl. 139 (1989), 586-599.

4. C. Miranda, Formule di maggiorazione e teorema di esistenza per le funzioni biarmoniche di due variabili, Giorn. Mat. Battaglini 78 (1948), 97-118.

5. P. W. Schaefer, On a maximum principle for a class of fourth order semilinear elliptic equations, Proc. Roy. Soc. Edinburgh Sect A. 77 (1977), 319-323.

6. P. W. Schaefer, Uniqueness in some higher order elliptic boundary value problems, Z. Angew. Math. Phys. 29 (1978), 693-697.

7. R. P. Sperb, Maximum principles and their applications (Academic Press, 1981).

8. S. Tseng and C.-S. Lin, On a subharmonic functional of some even order elliptic problems, J. Math. Anal. Appl. 207 (1997), 127-157.

9. H. Zhang and W. Zhang, Maximium principles and bounds in a class of fourth-order uniformly elliptic equations, J. Phys. A: Math. Gen. 35 (2002), 9245-9250. 